\title{
Appropriation of GPIb $\alpha$ from platelet-derived extracellular vesicles supports monocyte recruitment in systemic inflammation
}

Haematologica 2020

Volume 105(5):1248-1261

\section{Correspondence: \\ MYRIAM CHIMEN \\ chimenm@bham.ac.uk \\ Received: December 21, 2018. \\ Accepted: August 23, 2019. \\ Pre-published: August 29, 2019.}

doi:10.3324/haematol.2018.215145

Check the online version for the most updated information on this article, online supplements, and information on authorship \& disclosures: www. haematologica.org/content/105/5/1248

(C)2020 Ferrata Storti Foundation

Material published in Haematologica is covered by copyright. All rights are reserved to the Ferrata Storti Foundation. Use of published material is allowed under the following terms and conditions:

https://creativecommons.org/licenses/by-nc/4.0/legalcode. Copies of published material are allowed for personal or internal use. Sharing published material for non-commercial purposes is subject to the following conditions:

https://creativecommons.org/licenses/by-nc/4.0/legalcode, sect. 3. Reproducing and sharing published material for commercial purposes is not allowed without permission in writing from the publisher.

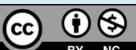

\begin{abstract}
Myriam Chimen, ${ }^{1,2}$ Aigli Evryviadou, ${ }^{1}$ Clare L. Box, ${ }^{1}$ Matthew J. Harrison, ${ }^{1}$ Jon Hazeldine, ${ }^{2}$ Lea H. Dib, ${ }^{1}$ Sahithi J. Kuravi, ${ }^{1}$ Holly Payne, ${ }^{1}$ Joshua M.J. Price, ${ }^{2}$ Dean Kavanagh, ${ }^{1}$ Asif J. Iqbal, ${ }^{1}$ Sian Lax, ${ }^{1}$ Neena Kalia, ${ }^{1}$ Alex Brill, $, 1,3,4$ Steve G. Thomas, ${ }^{1,3}$ Antonio Belli, ${ }^{2}$ Nicholas Crombie, ${ }^{2}$ Rachel A. Adams, ${ }^{5}$ Shelley-Ann Evans, ${ }^{5}$ Hans Deckmyn, ${ }^{6}$ Janet M. Lord, ${ }^{2}$ Paul Harrison, ${ }^{2}$ Steve P. Watson, ${ }^{1,3}$ Gerard B. Nash ${ }^{1}$ and G. Ed Rainger ${ }^{1}$
\end{abstract}

${ }^{1}$ Institute of Cardiovascular Sciences, College of Medicine and Dentistry, University of Birmingham, Birmingham, UK; ${ }^{2}$ NIHR Surgical Reconstruction and Microbiology Research Centre, Institute of Inflammation and Ageing, Birmingham University Medical School, Birmingham, UK; ${ }^{3}$ Centre of Membrane Proteins and Receptors, University of Birmingham and Nottingham, The Midlands, UK; ${ }^{4}$ Sechenov First Moscow State Medical University (Sechenov University), Moscow, Russia; ${ }^{5}$ Cardiff School of Health Sciences, Cardiff Metropolitan University, Cardiff, UK and ${ }^{6}$ Laboratory for Thrombosis Research, KU Leuven Campus Kulak Kortrijk, Kortrijk, Belgium

\section{ABSTRACT}

$\tau$ r nteractions between platelets, leukocytes and the vessel wall provide alternative pathological routes of thrombo-inflammatory leukocyte recruitment. We found that when platelets were activated by a range of agonists in whole blood, they shed platelet-derived extracellular vesicles which rapidly and preferentially bound to blood monocytes compared to other leukocytes. Platelet-derived extracellular vesicle binding to monocytes was initiated by P-selectin-dependent adhesion and was stabilised by binding of phosphatidylserine. These interactions resulted in the progressive transfer of the platelet adhesion receptor GPIb $\alpha$ to monocytes. GPIb $\alpha^{+}$monocytes tethered and rolled on immobilised von Willebrand Factor or were recruited and activated on endothelial cells treated with TGF- $\beta 1$ to induce the expression of von Willebrand Factor. In both models monocyte adhesion was ablated by a function-blocking antibody against GPIb $\alpha$. Monocytes could also bind platelet-derived extracellular vesicle in mouse blood in vitro and in vivo. Intratracheal instillations of diesel nanoparticles, to model chronic pulmonary inflammation, induced accumulation of GPIb $\alpha$ on circulating monocytes. In intravital experiments, GPIb $\alpha^{+}$-monocytes adhered to the microcirculation of the TGF- $\beta 1$-stimulated cremaster muscle, while in the $\mathrm{ApoE}^{-1}$ model of atherosclerosis, GPIb $\alpha^{+}$-monocytes adhered to the carotid arteries. In trauma patients, monocytes bore platelet markers within 1 hour of injury, the levels of which correlated with severity of trauma and resulted in monocyte clearance from the circulation. Thus, we have defined a novel thrombo-inflammatory pathway in which platelet-derived extracellular vesicles transfer a platelet adhesion receptor to monocytes, allowing their recruitment in large and small blood vessels, and which is likely to be pathogenic.

\section{Introduction}

The recruitment of leukocytes during inflammation occurs in the haemodynamically permissive environment of the post capillary venules. In this environment, vascular endothelial cells responding to pro-inflammatory mediators such as cytokines express adhesion receptors and activating stimuli such as chemokines, which ensure efficient and localised trafficking of leukocytes into the affected tissues. ${ }^{1-4}$ It has become clear more recently that in pathological situations, platelets can also play a 
role in leukocyte recruitment in other vascular beds. ${ }^{5}$ Thus, the integrated function of the thrombotic and inflammatory systems results in recruitment of leukocytes to arterioles in models of ischaemic injury of the liver and other tissues. ${ }^{6-10}$ Moreover, there is substantial evidence supporting a role for platelets in the preferential recruitment of monocytes to the artery wall during atherogenesis. For example, inhibition of platelet adhesion to the artery wall, or induction of thrombocytopenia, significantly reduces monocyte trafficking and the burden of atherosclerotic disease in genetically susceptible strains of mice. ${ }^{11-14}$ In addition, instillation of activated platelets exacerbates the formation of atherosclerotic plaques in such models. ${ }^{11-14}$ There is also direct evidence that platelet P-selectin plays a role in plaque formation in the ApoE $E^{-/}$mouse. ${ }^{11-14}$ Other studies demonstrate that platelet derived chemokines such as CCL5 (RANTES) and CX3CL1 (fractalkine), once deposited on vascular endothelial cells, can selectively recruit monocytes in these models. ${ }^{11-15}$

The examples described above require platelet activation at the vessel wall to facilitate leukocyte recruitment and trafficking. However, interactions between platelets and leukocytes also occur in circulating blood under pathological conditions. Indeed, formation of plateletleukocyte aggregates has been described in diseases as diverse as bacterial infection, rheumatoid arthritis, diabetes and inflammatory bowel disease. ${ }^{16-22}$ In cardiovascular disease (CVD) the number of platelet-leukocyte aggregates increases significantly, and one can measure an increased incidence of such heterotypic aggregates in individuals with independent risk factors for CVD, such as hypertension..$^{23-25}$ Indeed, it has been proposed that an increase in the incidence of platelet-leukocyte aggregates may in itself, be an independent risk factor for CVD. ${ }^{26} \mathrm{The}$ formation of platelet leukocyte aggregates may also play an important role in acute and severe inflammatory responses. Thus, in patients with acute trauma or trauma associated sepsis, an enhanced capacity for platelet activation and platelet interaction with monocytes and neutrophils has been reported in response to exogenous activation of their blood with the ionophore, ionomycin. ${ }^{27,28}$

Extracellular vesicles which can be detected in the blood, urine and other bodily fluids are heterogeneous particles 40-1,500 $\mathrm{nm}$ in diameter that are derived from the plasma membrane (microvesicles) or by exocytosis of multi-vesicular bodies (exosomes). ${ }^{29}$ They are released from cells of the vasculature, including platelets, endothelial cells (EC) and leukocytes, and specific populations can be identified using appropriate methodology (e.g. flow cytometry), as they express surface markers derived from their cell of origin. There is now mounting evidence that platelet-derived extracellular vesicles (PEV) (otherwise and often referred to as microparticles or microvesicles) are heterogeneous in nature. For example, in vitro, PEV have been generated in response to shear stress, thrombin, calcium ionophore, adenosine diphosphate (ADP), collagen and collagen related peptide. ${ }^{30-33}$ Interestingly, these studies show that PEV derive by using different platelet agonists and differ in abundance, as well as the cargo that they convey. Indeed, there is now good evidence that platelets can shed large vesicles which contain organelles such as mitochondria. ${ }^{34}$ Until recent technological advancements it had been impossible to analyse the concentration and composition of vesicles using a single platform. Flow cytometry does not detect vesicles $<200$ -
$300 \mathrm{~nm}$ and does not accurately measure larger vesicles due to the disparity in the refractive index of biological vesicles and the latex beads used as size standards on this platform. ${ }^{35}$ However, electron microscopy studies show that the majority of PEV are small. Thus, although Ponomereva et al. described calcium ionophore derived $\mathrm{PEV}$ as large as $1,500 \mathrm{~nm}$, particles were predominantly in the range of 50-130 nm. ${ }^{36}$ Similarly, Aatonen et al. described the main population of PEV as being 100-250 $\mathrm{nm}$, with in excess of $90 \%$ of all vesicles being smaller than $500 \mathrm{~nm}$ irrespective of the platelet agonist used for PEV biogenesis. ${ }^{35}$ Mitochondria containing vesicles, referred to above, were in the range of 500-1,500 nm. Importantly, the study of the functions of distinct subsets of PEV is not a well-developed field, however, bearing in mind the diversity of the PEV generated upon platelet activation, vesicles with discrete functional roles cannot be ruled out. The diversity of platelet microparticles has recently been reviewed. ${ }^{37}$

There is mounting evidence that PEV play a pathophysiological role in inflammation. ${ }^{38}$ An increased concentration of circulating PEV is associated with a number of diseases. In diabetic retinopathy, the number of PEV was associated with the severity of disease, ${ }^{39}$ while the levels of PEV circulating in patients with type-1 diabetes correlated with the degree of pro-atherogenic dyslipidaemia. ${ }^{40}$ There was a correlation with vascular dysfunction (assessed by measuring arterial elasticity and flow-dependent vasodilatation of the brachial artery) in patients with type- 2 diabetes. ${ }^{41}$ Interestingly, the number of PEV was higher in patients with acute coronary syndromes than those with stable angina, ${ }^{42}$ implying an association with the onset of athero-thrombotic disease. The roles of PEV in inflammation and pathogenesis of inflammatory disease are not well understood. However, they possess adhesion receptors such as glycoprotein (GP)Ib $\alpha$, $\alpha \operatorname{Ilb} \beta 3$ integrin and P-selectin, meaning that they could interact with the vessel wall and circulating leukocytes to promote recruitment of the later. Importantly, as these receptors ordinarily regulate the process of haemostasis, PEV might provide an avenue of leukocyte recruitment to the disease environment which falls outside of regulatory pathways which ordinarily limit the duration and magnitude of the inflammatory response.

Here, using assays of heterotypic aggregate formation we have characterised the adhesive interactions between leukocytes and PEV in whole blood and identified a novel route by which the platelet adhesion receptor, GPIb $\alpha$, promotes monocyte recruitment in both in vitro and in vivo models of vascular inflammation.

\section{Methods}

Full Methods can be found in the Online Supplementary Materials and Methods.

\section{Blood donors}

Blood was obtained from healthy donors with informed consent and with local ethical approval (ERN_07-058). Blood from the Golden Hour cohort (drawn within 1 hour of suffering traumatic injury) was obtained under the National Research Ethics Committee (reference 13/WA/0399). Specimen collection and informed consent procedures were approved and permission granted by the Biomedical Science Ethic Committee. 


\section{Animal experiments}

All experiments were performed in accordance with the Home Office Guidelines. In each experiment C57BL/6 IL4R/GPIb $\alpha-\mathrm{Tg}$ or $A p o E^{--}$or wild-type (WT) animals with the same background were allocated at random to experimental groups. Mice from the same litter were randomly distributed amongst experimental groups.

\section{Results}

Platelet activation in whole blood leads to formation of PEV and their adhesion to monocytes

We investigated the effect of platelet activation on platelet-leukocyte interactions in whole blood. When thrombin receptor activating peptide (TRAP), an agonist of the platelet protease activated receptor-1 (PAR-1), was added to sheared whole blood, a time dependent increase in the percentage of monocytes bearing the platelet receptor GPIba (CD42b) as well as CD41 (GPIIb) and in the intensity of GPIb $\alpha$ and CD41 staining, was observed (Figure 1A-C; Online Supplementary Figure S1A-C and S2). In unstimulated blood, few monocytes $(\sim 5 \%)$ possessed measurable levels of GPIbo, showing that shear did not activate platelets. During analysis monocytes were subdivided into two subsets using standard markers as previously described. ${ }^{43}$ Classical monocytes $\left(\mathrm{CD} 14^{+} \mathrm{CD} 16^{-}\right)$represent $90 \%$ of cells in the circulation and nonclassical/intermediate monocytes $\left(\mathrm{CD} 14^{+/ \mathrm{dim}} \mathrm{CD} 16^{+}\right) 10 \%$ (Online Supplementary Figure S1A). In our studies, we have compared classical to non-classical/intermediate monocytes grouped together. This is because the low numbers of isolated intermediate and non-classical monocytes do not allow appropriate functional testing of these subsets individually in our assays. The interaction between platelets and monocytes was similar when classical and non-classical/intermediate monocytes were assayed, showing similar patterns of GPIb $\alpha$ and CD41 accumulation over time (Figure 1B-C; Online Supplementary Figure S1A-C and S2). Interestingly, only a modest accumulation of GPIb $\alpha$ was evident on neutrophils stimulated with TRAP and even less when whole blood was stimulated with CRP-XL (Figure 1D and Online Supplementary Figure $S 1 D-E)$. We observed no accumulation of GPIba on lymphocytes (Figure 1D and Online Supplementary Figure S1D).

The median fluorescent intensity (MFI) of GPIba on monocytes after 30 minutes (min) of TRAP stimulation was well below the intensity on individual platelets (Figure 2A). Moreover, the time course of the acquisition of GPIb $\alpha$ by monocytes demonstrated a progressive accumulation that ruled out the binding of whole platelets (Figure 2B). This pattern of accumulation is consistent with the adhesion of PEV, which was confirmed using confocal microscopy (Figure 2C and Online Supplementary Figure S3). For comparison, we show a monocyte bearing whole platelets generated under different experimental conditions i.e. in the absence of shear (Figure 2D).

Here, we have reported the formation of $\mathrm{PEV}$ in response to thromboxane $\mathrm{A} 2, \mathrm{ADP}$ and cross linked collagen related peptide (CRP-XL). Activation of platelets in whole blood using CRP-XL, ADP, the thromboxane mimetic U46619, or the C-type lectin-like receptor (CLEC-2) agonist, rhodocytin, resulted in the same pattern of accumulation of GPIb $\alpha$ on monocytes, showing that different routes of platelet activation resulted in PEV pro- duction and adhesion to monocytes (Online Supplementary Figure $S 4 A-D)$.

\section{PEV binding to monocytes is rapid}

Accumulation of PEV on monocytes after stimulation of whole blood was progressive over a prolonged period of time (i.e. $30 \mathrm{~min}$ ) (Figure $1 \mathrm{~B}-\mathrm{C}$ and Online Supplementary Figure S4). An important question is whether this pattern of accumulation is dependent upon the dynamics of PEVmonocyte interaction and adhesion, or whether the genesis of PEV from activated platelets is the rate-limiting step. Here we used the addition of isolated and prelabelled PEV $\left(1 \times 10^{9} / \mathrm{mL}\right)$ generated by stimulating platelets $(3 \times 108)$ with CRP-XL $(1 \mu \mathrm{g} / \mathrm{mL})$, to unstimulated whole blood to investigate this. After CRP-XL activation of isolated platelets, GPIb $\alpha$ stained PEV were readily discernible by flow cytometry in platelet supernatants (Figure 3A and Online Supplementary Figure S5A-E). A similar pattern was observed for CD41 on PEV (Online Supplementary Figure S5C and S6). Interestingly, 25\% of the large vesicles detected by flow cytometry contained mitochondrial fragments, as previously described ${ }^{34}$ (Online Supplementary Figure S7A). Analysis using nanoparticle tracking showed that $3 \times 10^{8}$ platelets could yield $1.2 \pm 0.3 \times 10^{9} \mathrm{PEV}$ compared to an average $1.3 \times 10^{8} \pm 2.8 \times 10^{7}$ vesicles in untreated conditions (Figure $3 \mathrm{~B}$ and Online Supplementary Figure S5D-E) with a mean diameter of $274 \pm 188 \mathrm{~nm}$. To date it has not been possible to simultaneously count vesicles, size them and analyse protein cargo using a single platform. The Exo View-R100 is a new platform which allows such analysis providing previously unattainable information in a single protocol. ${ }^{44}$ Using this assay we observed that PEV from CRP-XL stimulated platelets captured by a CD9 antibody had a mean size of $54 \mathrm{~nm}$ while those captured by a CD41a antibody had a mean size of $82.3 \mathrm{~nm}$ (Online Supplementary Figure S7B). Upon analysis using the Exo View system, we observed the majority of PEV were captured by CD41a and CD9 (which are abundant on platelets), but not CD63 or CD81 (which are expressed on exosomes) (Online Supplementary Figure S7C). This was also confirmed by secondary labelling of captured PEV using fluorescent antibodies against CD9, CD63 and CD81 (Online Supplementary Figure S7D). Labelled PEV were added to whole blood at a concentration of $1 \times 10 \% / \mathrm{mL}$ and their interactions with leukocytes assayed by flow cytometry. Many monocytes acquired GPIbo within $5 \mathrm{~min}$, but neutrophils or lymphocytes did not (Figure 3C-D). The proportion of monocytes acquiring GPIbo slowly increased thereafter, while intensity of GPIb $\alpha$ staining increased steadily (Figure 3C-D). Interestingly, we found that most of the GPIbo signal detected by flow cytometry was intracellular ( $80 \%)$ on both monocyte subsets (Online Supplementary Figure S8A$B)$ and in agreement with the confocal imaging data described in Figure 2C. We also analysed the adhesion of PEV labelled with the lipophilic dye PKH67 to exclude antibody-mediated interaction of PEV with monocytes. PKH67 labelled all of the PEV in the activated-platelet supernatant (Figure $3 \mathrm{E}$ ). The dynamics of PEV binding to monocytes, neutrophils or lymphocytes (Figure 3F) was similar to that for the antibody-labelled PEV (Figure 3C).

The mechanistic basis for the preferential accumulation of GPIb $\alpha$ on monocytes was investigated using adhesionblocking reagents. Inclusion of a function-neutralising 
anti-P-selectin antibody inhibited GPIb $\alpha$ accumulation on both monocytes and neutrophils, strongly implicating this platelet receptor in heterotypic adhesion with the two cells (Figure 4A and Online Supplementary Figure S9A$B)$. We measured the density of the P-selectin counter receptor P-selectin Glycoprotein Ligand 1 (PSGL-1) on blood leukocytes because the efficiency of GPIb $\alpha$ accumulation might reflect the surface density of this molecule. Figure $4 \mathrm{~B}$ shows that there is substantially more PSGL-1 on monocytes than neutrophils, which could account for the differential levels of GPIb $\alpha$ accumulating on these cells. However, T cells, which did not accumulate GPIb $\alpha$, also possessed abundant PSGL-1. Thus additional and cell specific adhesive interactions may be required to stabilise $\mathrm{P}$-selectin mediated adhesion under shear. Using a panel of function-neutralising antibodies against known platelet and leukocyte adhesion molecules, we could find no contribution to heterotypic aggregate formation from CD31, ICAM-2 or $\beta 2$-integrins (Figure 4C-E). However, an anti-phosphatidylserine (PS) antibody significantly reduced GPIb $\alpha$ accumulation on both neutrophils and monocytes (Figure 4C-E). The function of PS as a stabilising interactant is concordant with its documented patterns of interaction with monocytes and neutrophils, while its potential lack of interaction with T cells would account for the lack of GPIb $\alpha$ accumulation on these cells.

An important question was whether monocyte activa-

A

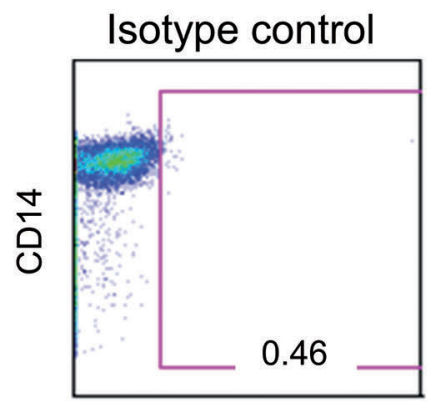

GPIba

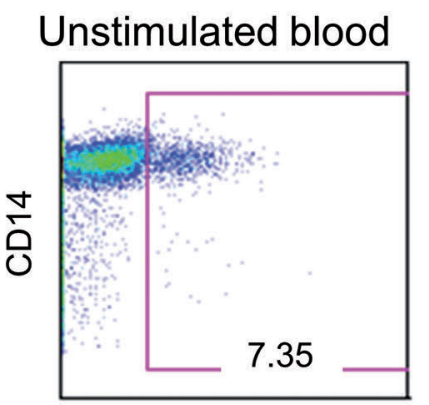

GPlba

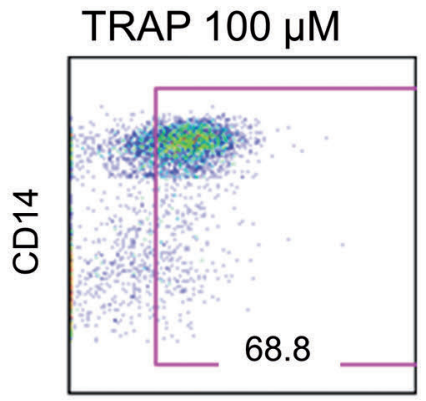

GPIba
B

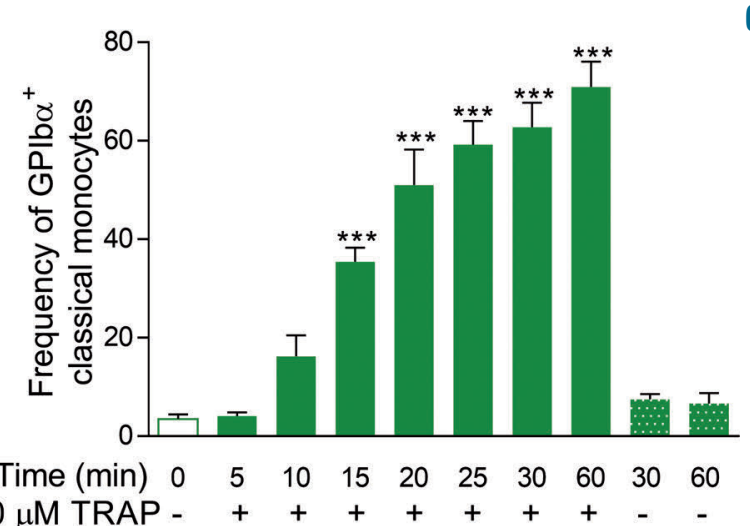

C

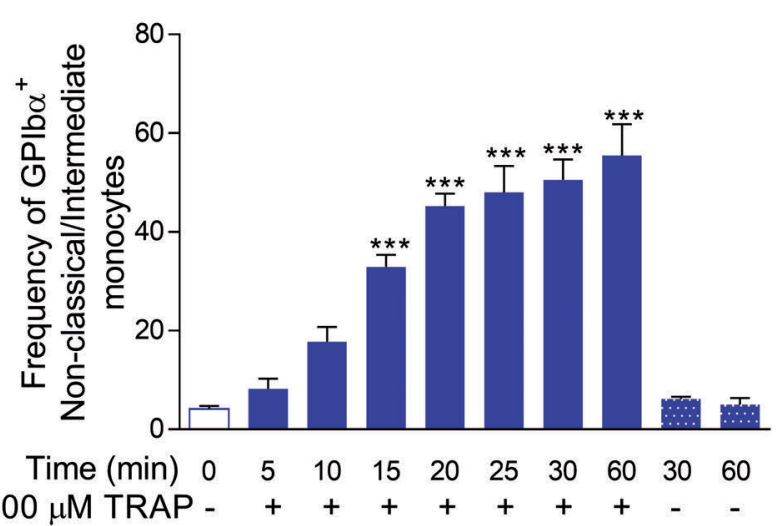

D

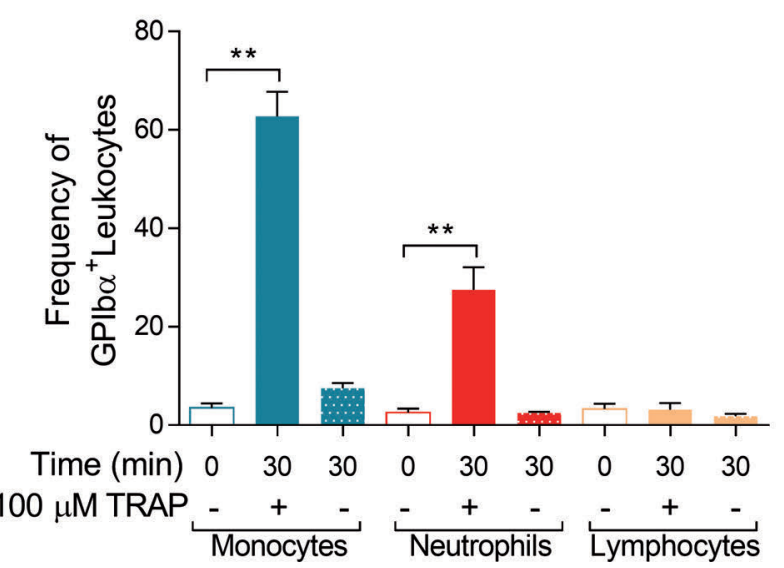

Figure 1. Accumulation of GPIb $\alpha$ on leukocytes in stimulated whole blood. (A) Representative plots of GPIb $\alpha$ labelling on monocytes in whole blood unstimulated or stimulated with TRAP $(100 \mu \mathrm{M})$ for 60 minutes ( $\mathrm{min}$ ) under shear measured by flow cytometry. (B-D) Percentage of GPIb $\alpha^{+}$classical monocytes (B), non-classi$\mathrm{cal} /$ intermediate monocytes (C), and monocytes (all), lymphocytes and neutrophils (D) in whole blood stimulated with $100 \mathrm{MM}$ TRAP at $37^{\circ} \mathrm{C}$ under shear for 0 to 60 $\min , n=3$. Data are mean \pm standard error of the mean (SEM). ${ }^{* * P} \leq 0.01, * * * P \leq 0.001$ compared to the $0 \mathrm{~min}$ control (B, C, D) by analysis of variance (ANOVA) and Dunnett post-test. 
tion contributed to GPIb $\alpha$ accumulation. For these studies, we used CRP-XL to stimulate whole blood, as this agonist does not directly activate monocytes and would thus allow analysis of whether secondary activation of monocytes downstream of platelet activation was prerequisite for PEV adhesion. We assessed the expression of the activation marker $\alpha M \beta 2$-integrin (CD11b/CD18) on monocytes $30 \mathrm{~min}$ after the addition of CRP-XL to whole blood. There was some increase in both integrin subunits CD11b and CD18 (Online Supplementary Figure S9C-D), however, this was inconsistent and monocyte subset specific. When a function neutralising antibody against CD18 was included in the assay it had no effect on GPIb $\alpha$ accumulation (Figure 4C-E), indicating that monocyte activation was not required for PEV adhesion.

\section{Adopted GPIb $\alpha$ is a functional adhesion molecule} supporting monocyte rolling on von Willebrand Factor

As GPIb $\alpha$ is known to mediate binding of platelets from flowing blood to von Willebrand Factor (VWF), we tested whether VWF could also recruit PEV-treated monocytes (Figure 5A-E). Monocytes lacking GPIb $\alpha$ showed low levels of adhesion when perfused across immobilised human VWF (Figure 5B, E). However, acquisition of PEVderived-GPIb $\alpha$ supported capture and rolling $(66.8 \pm 4.1 \%$ of adherent cells rolling) of monocytes on VWF (Figure 5C, E). Importantly, the adhesion of PEV-treated mono- cytes was inhibited by a function-neutralising antibody against GPIb $\alpha$ (Figure 5D, E).

\section{Monocytes bearing GPIb $\alpha$ bind to EC in a model of vascular inflammation}

Transforming growth factor beta-1 (TGF- $\beta 1$ ) promotes the expression of a matrix of VWF on the surface of EC which recruits platelets from flowing blood, which in turn function as adhesive bridges for the preferential recruitment of monocytes to EC in vitro and in vivo. ${ }^{15}$ Here we used this model to determine whether PEV-derived GPIba could support monocyte adhesion directly to stimulated endothelium. A low level of monocyte adhesion to TGF- $\beta 1$-stimulated EC was observed without PEV (Figure 6A, D). However, PEV-treated monocytes adhered in significantly higher numbers, an adhesive interaction blocked by a GPIb $\alpha$ blocking antibody (Figure 6B-D). As previously observed, recruited monocytes did not roll on the EC. Thus $6.1 \pm 0.9 \%$ of adherent cells were observed rolling, with the remaining $93.9 \%$ becoming activated and stably adherent. Interestingly, the acquisition of PEV increased the efficiency with which monocytes transmigrated across the EC monolayer (Figure 6E). We could attribute this increase in PEVtreated monocytes recruitment to PEV rather than soluble factors such as chemokines, as supernatants generated from PEV filtered using a $10 \mathrm{KDa}$ size filter (to remove
A

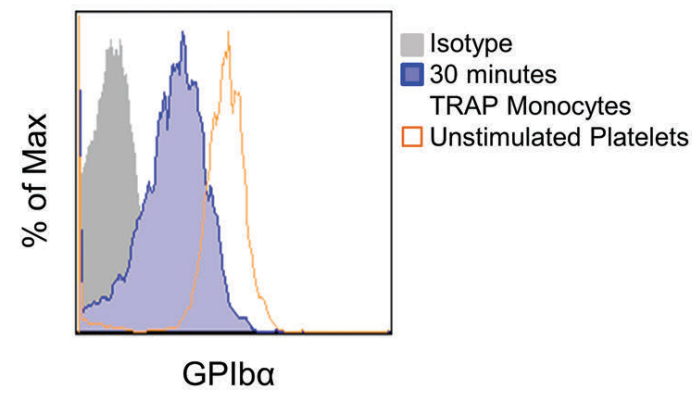

C

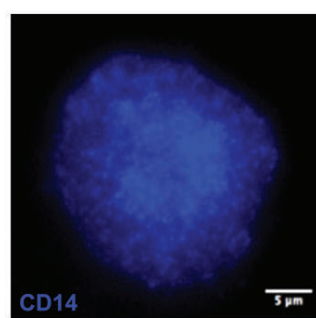

Merge
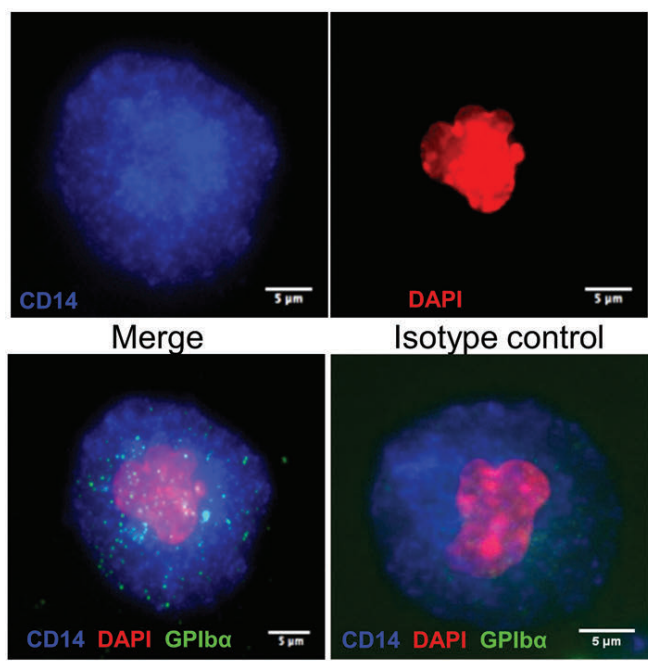

Isotype control

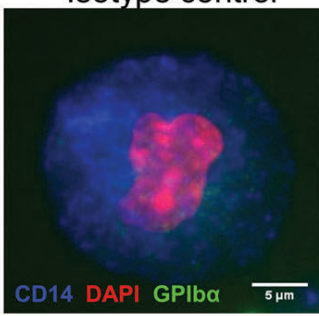

B

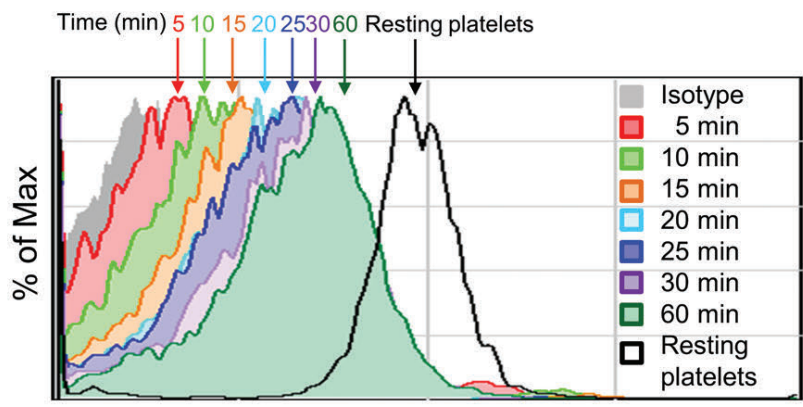

GPIba

D
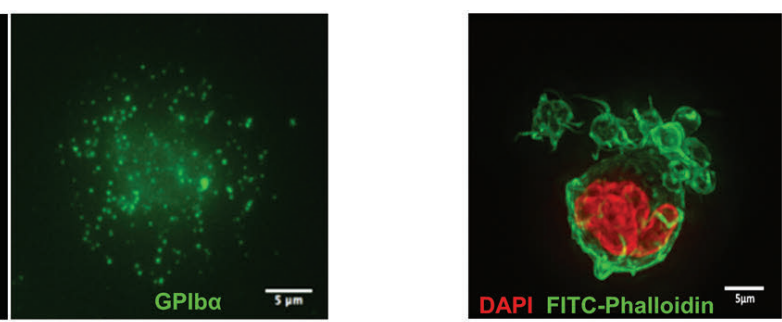

Figure 2. GPIb $\alpha$ on monocytes in stimulated whole blood derives from plateletderived extracellular vesicles. (A, B) Representative plots of GPIb $\alpha$ labelling on monocytes (all) in unstimulated or TRAP $(100 \mu \mathrm{M})$-stimulated whole blood under shear for 30 minutes (min) (A) and over time (B) measured by flow cytometry. (C) Representative pictures of monocytes labelled with anti-CD14, anti-GPIb $\alpha$ for platelet-derived extracellular vesicles (PEV) and DAPI imaged by confocal microscopy. Monocytes were isolated and incubated with CRP-XL-generated PEV for $30 \mathrm{~min}$ at $37^{\circ} \mathrm{C}$ under shear prior fixing and labelling. (D) Monocytes bound to platelets, both labelled with FITC-phalloidin and DAPI imaged by confocal microscopy. 
vesicles) did not induce monocyte adhesion and transmigration (Figure 6D, E).

\section{PEV-treated murine monocytes bearing GPIb $\alpha$ can be generated and recruited in mice}

Prior to moving to in vivo assays of monocyte recruitment, we determined whether murine PEV derived-GPIb $\alpha$ could accumulate on murine monocytes. Using the ex vivo whole blood assay under shear, we observed a high proportion of murine monocytes rapidly accumulated GPIb $\alpha$ and CD41 after addition of ADP to the blood (Figure 7A and Online Supplementary Figure S10A-C). To examine
monocytes/PEV aggregate formation in vivo we induced pulmonary inflammation by instillation of air pollution particles into the lungs. A significant increase in the number of monocytes bearing GPIb $\alpha$ and CD41 ( $\alpha$ IIb-integrin) was observed in animals exposed to air pollution particles, but not vehicle control (PBS) (Figure 7B-C). Importantly, and in concordance with human studies, GPIb $\alpha$ and CD41 intensities of expression was below the level on individual platelets (Online Supplementary Figure S10D), demonstrating that monocytes bind PEV in this model.

Using an intravital preparation of the TGF- $\beta 1$-stimulated, mouse cremaster muscle to observe monocyte interactions with the microvasculature in real time, we tracked
A

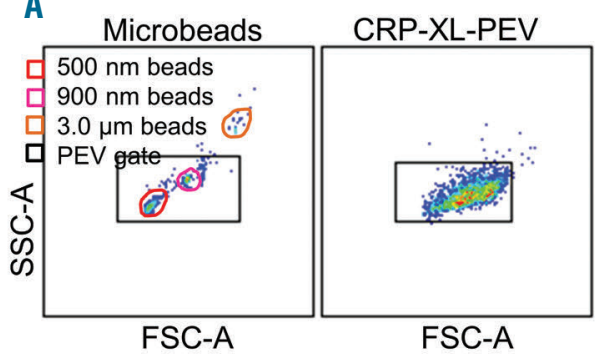

C

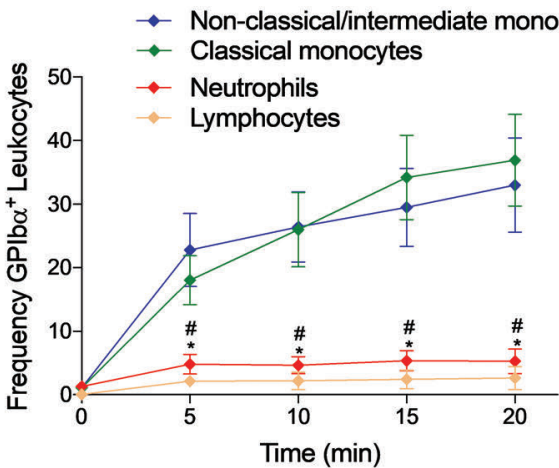

$E$

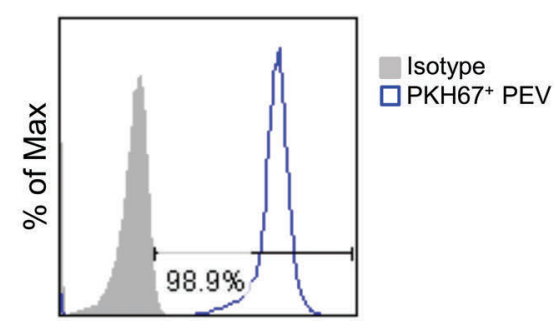

PKH67
B
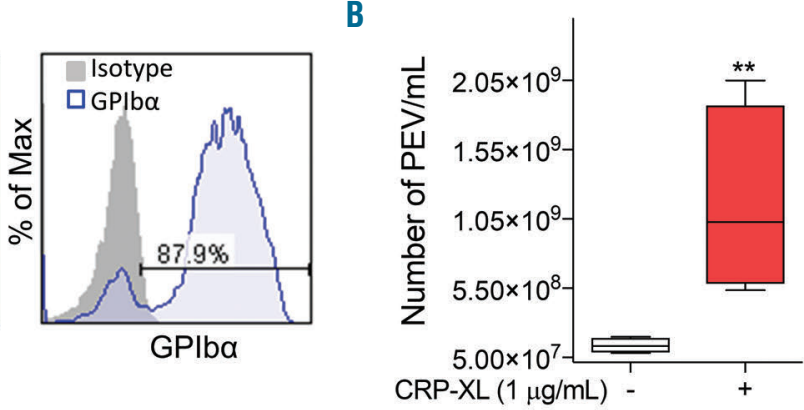

D
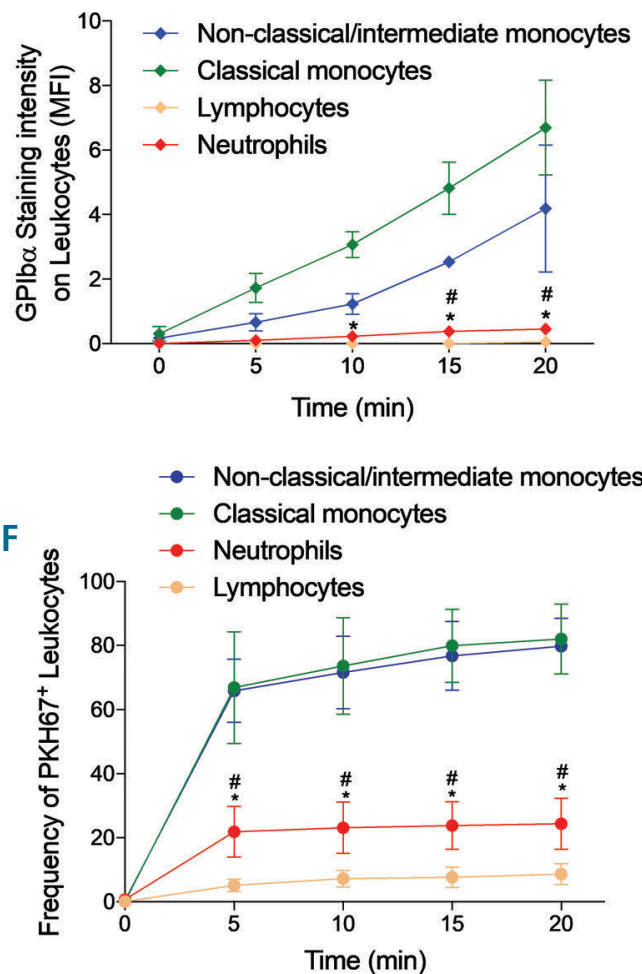

Figure 3. Rapid and specific binding of platelet-derived extracellular vesicles to monocytes. (A) Platelet-derived extracellular vesicles (PEV) gate was determined using microbeads to select events under $900 \mathrm{~nm}$ in size (left dot plots). Representative plot of GPIba intensity on PEV generated by stimulation of platelets with 1 $\mu \mathrm{g} / \mathrm{mL}$ CRP-XL for 30 minutes ( $\mathrm{min}$ ) analysed by flow cytometry (right histogram). (B) Concentration of PEV generated by stimulation of platelets with $1 \mu \mathrm{g} / \mathrm{mL} \mathrm{CRP}$ $\mathrm{XL}$ for $30 \mathrm{~min}, \mathrm{n}=4$. (C, D) Percentage (C) and median fluorescent intensity (MFI) (D) of GPIb $\alpha^{+}$leukocytes in unstimulated whole blood supplemented with CRP_XL $\left(1 \mu \mathrm{g} / \mathrm{mL}\right.$ )-generated-PEV at $37^{\circ} \mathrm{C}$ under shear determined by flow cytometry, $\mathrm{n}=4$. (E) Representative plot of PEV generated by stimulation of platelets with $1 \mathrm{\mu g} / \mathrm{mL}$ CRP-XL for 30 min labelled with PKH67. (F) Percentage of PKH67+ leukocytes in unstimulated whole blood supplemented with CRP_XL (1 $\mu$ g/mL) generated PEV at

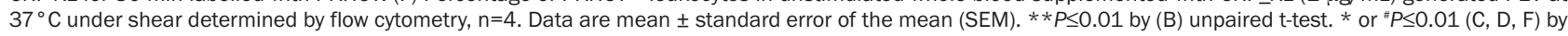
repeated measures two-way ANOVA followed by Bonferroni post-test for neutrophils and lymphocytes compared to * classical monocytes and \# to nonclassical/Intermediate monocytes. 
human mouse PEV-treated monocytes (Online Supplementary Figure S1OE-F). We used the hIL4R/GPIba-Tg mouse which expressed human IL-4 receptor under the GPIb $\alpha$ promoter. This allows the animals to be rendered thrombocytopenic by injection of an antibody against hIL4R. Adoptively transferred WT platelets or PEV are however retained within the circulation. Using mice depleted of endogenous platelets using an anti-hIL4R antibody, we observed higher numbers of adoptively transferred WT PEV-treated monocytes rolling on the microvasculature compared to untreated monocytes; the number was significantly reduced by a GPIb $\alpha$ blocking antibody (Figure 7D-G). Detailed analysis revealed two populations of rolling cells: those exhibiting stable rolling (interactions $>300 \mathrm{~ms}$ ) with a velocity of $241 \pm 82 \mu \mathrm{m} / \mathrm{s}$ (Figure 7D, F); those exhibiting transient rolling (interactions $<300 \mathrm{~ms}$ ) with a velocity of $478 \pm 65$ $\mu \mathrm{m} / \mathrm{s}$ (Figure 7D, G). We also infused human monocytes into $A p o E^{-r}$ mice that had been on a western diet for six weeks and observed the carotid artery by intravital microscopy. Murine PEV-treated monocytes adhered to the artery wall with significantly greater efficiency than untreated monocytes (Figure $7 \mathrm{H}$ ). In this environment a mixture of adhesive behaviors was observed with stationary adhesion, stable rolling and transient rolling adhesion evident (Figure 7H).

\section{Monocytes with platelet markers appear within 1 hour of severe trauma and are rapidly cleared from the circulation}

We investigated whether rapid production and binding of extracellular vesicles to monocytes could be detected following an acute event such as traumatic injury. In the Golden Hour study blood samples in the pre-hospital setting (mean time to blood sampling $=43 \mathrm{~min}$ ) were acquired from traumatically-injured patients (injury sever-
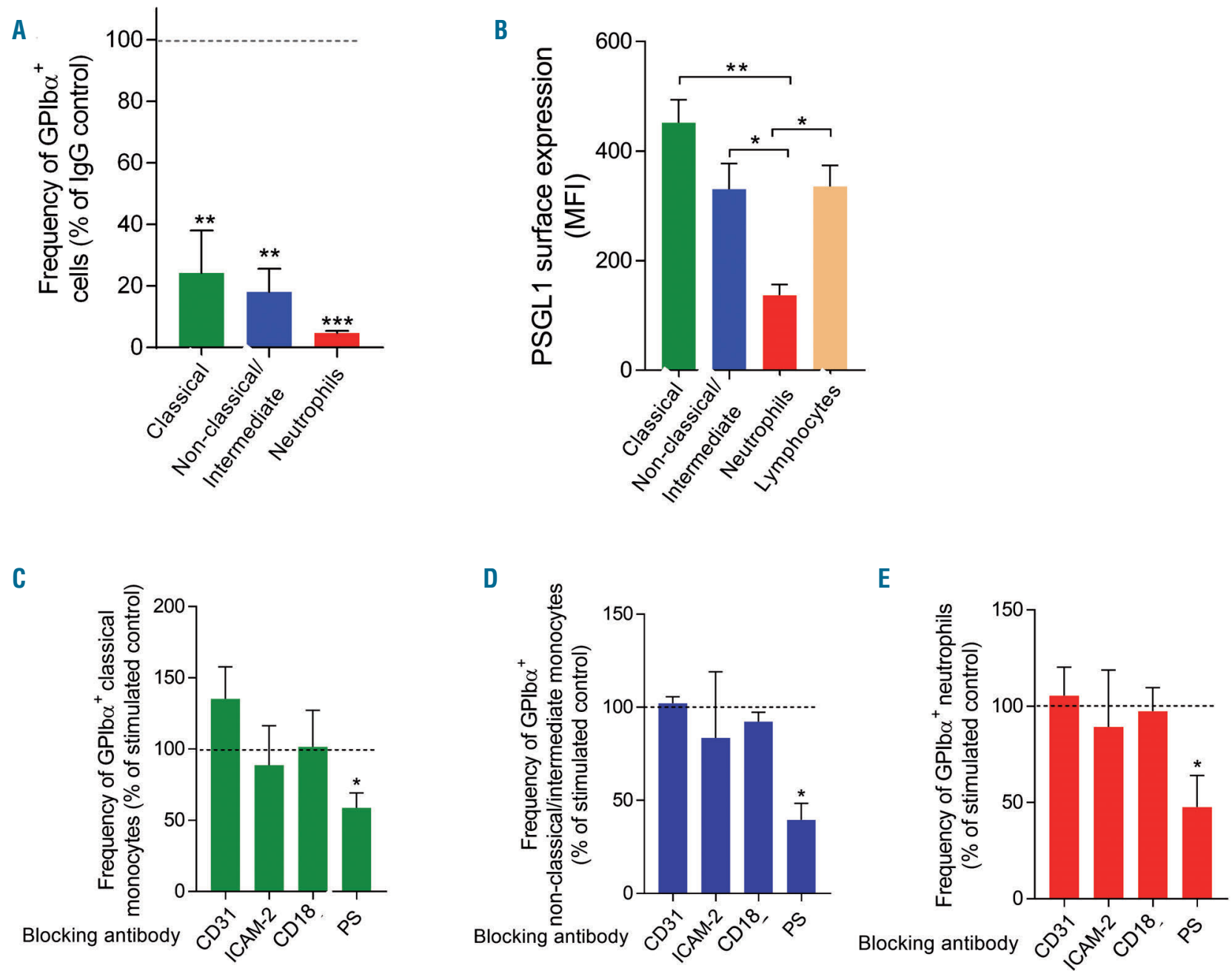

Figure 4. Blocking of GPIba $\alpha^{+}$platelet-derived extracellular vesicles to leukocytes. (A) Binding of platelet-derived extracellular vesicles (PEV) on classical monocytes, non-classical/intermediate monocytes and neutrophils with blockade of P-selectin in TRAP $(100 \mu \mathrm{M})$-stimulated whole blood for 30 minutes (min) under shear, $\mathrm{n}=3$. (B) Surface expression (MFI: median fluorescence intensity) of PSGL1 (P-selectin ligand) on monocyte subsets, neutrophils and lymphocytes determined by flow cytometry, $n=3$. (C-E) Binding of PEV on classical monocytes (C), non-classical/intermediate monocytes (D) and neutrophils (E) with blockade of CD31, ICAM-2, CD18 $(\beta 2)$ and Phosphatidylserine (PS) in TRAP $(100 \mu \mathrm{M})$-stimulated whole blood for 30 min under shear, $n=3-5$. Data are mean \pm standard error of the mean (SEM). $* P \leq 0.05, * * P \leq 0.01$ compared the normalised IgG control (A) by analysis of variance (ANOVA) and Dunnett post-test or Bonferroni post-test (B) and one sample $t$-test to $100 \%$ of TRAP control (C-E). 
ity score $[\mathrm{ISS}]>8$ ). Analysis by flow cytometry showed acquisition of CD41 by circulating leukocytes with preferential binding to monocytes (Figure 8A-B and Online Supplementary Figure S11A-B). The CD41 measured on monocytes was likely derived from PEV, as the intensity of fluorescent staining at 4 hours post trauma $(2,226 \pm 474)$ was substantially below that of a single platelet $(13,702 \pm 964)$ (Online Supplementary Figure S11B). Both the number of $\mathrm{CD} 41^{+}$monocytes and the intensity of staining for CD41 on them (MFI), correlated significantly with the severity of trauma (Figure 8C-D). Lastly, there was a marked loss of $\mathrm{CD} 41^{+}$monocytes from the blood within 4 to 12 hours, which was sustained for up to 72 hours (Figure 8E) and a decrease in circulating platelet counts which reflects platelet activation and PEV generation (Online Supplementary Figure S11C).

\section{Discussion}

We have defined a new thrombo-inflammatory route of monocyte recruitment via an adhesion molecule transferred from platelets. Recruitment is reliant upon plateletderived GPIb $\alpha$, which allows monocyte capture by VWF exposed on the vessel wall. Previous studies have indicated that platelet-derived chemokines can then induce arrest and migration. ${ }^{45}$ Online Supplementary Figure S12 summarises the steps we propose in this thrombo-inflammatory cascade. Importantly, the cascade may diverge from the normal pathways of leukocyte trafficking in a manner that could contribute to disease, as plasma borne PEV preferentially deliver functional GPIb $\alpha$ to the monocyte surface. Transfer of GPIb $\alpha$ can support adhesion of monocytes in vitro and in vivo, in human and murine models of

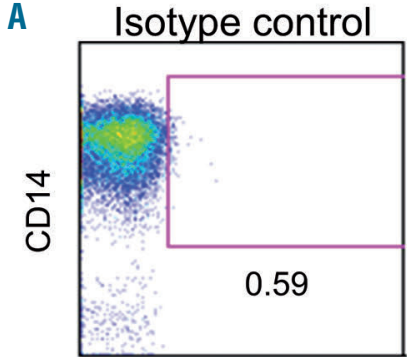

GPIba

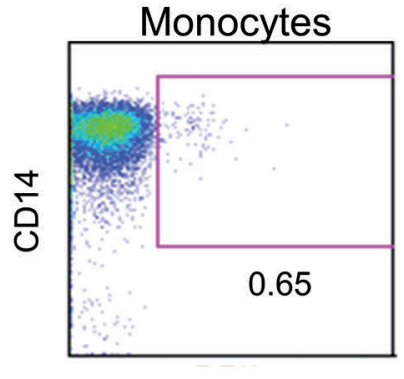

GPIba

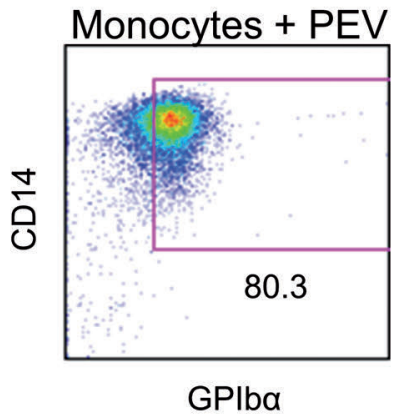

D
B

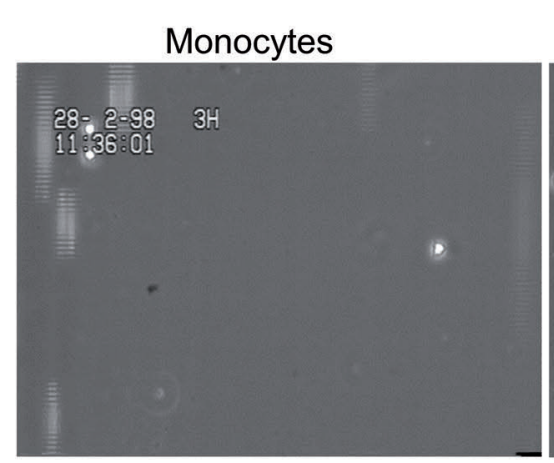

C

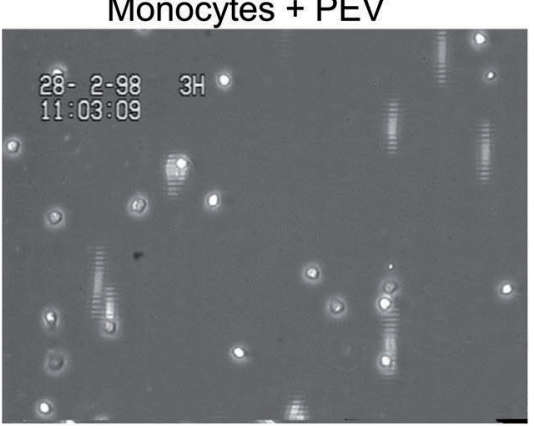

Monocytes+ PEV+ Anti-GPIba

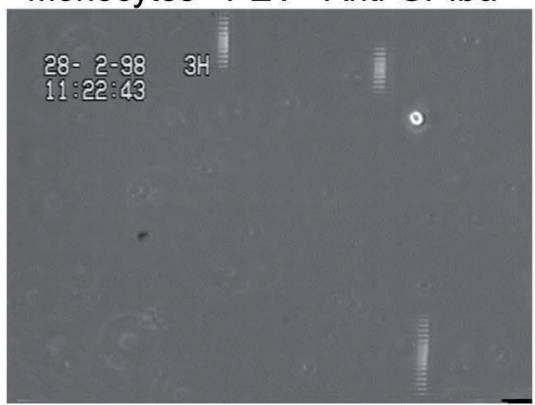

E

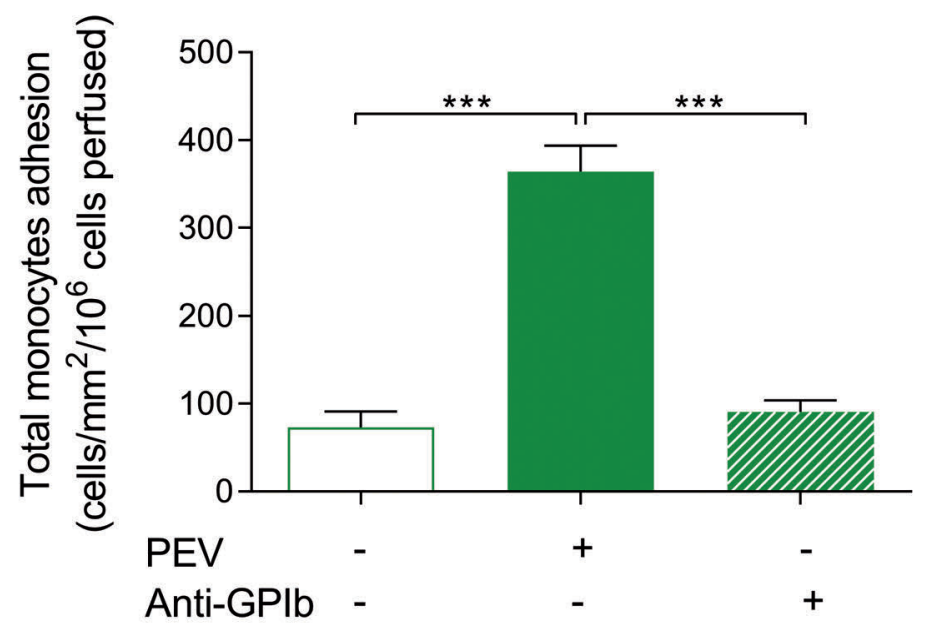

Figure 5. GPIb $\alpha$ derived from platelet-derived extracellular vesicles supports monocytes rolling on von WIIlebrand Factor. (A) Representative plots of CRP_XL $(1 \mu \mathrm{g} / \mathrm{mL})$ generated-GPIb $\alpha^{+}$plateletderived extracellular vesicles (PEV) bound to monocytes measured by flow cytometry. (B-D) Representative pictures of monocytes (B), monocytes bearing CRP_XL $(1 \mu \mathrm{g} / \mathrm{mL})$ generated-PEV (C) and monocytes bearing PEV with GPIb $\alpha$ blockade (clone 6B4, $20 \mu \mathrm{g} / \mathrm{mL}$ ) (D) recruited on von Willebrand Factor (VWF) under flow conditions. (E) Total adhesion of monocytes with or without PEV and GPIb $\alpha$ blockade on VWF in flow conditions, $n>10$. Data are mean \pm standard error of the mean (SEM). $* * P \leq 0.01$ by ANOVA and Bonferroni post-test. 
vascular inflammation. This process means that circulating monocytes may be recruited to the vessel wall through a pathway outside of the tightly regulated physiological inflammatory system. We believe that such monocyte recruitment may be particularly relevant in the dysregulated inflammatory responses seen in chronic inflammatory disease, which leads to tissue damage and loss of function (such as atherosclerosis and rheumatoid arthritis). In addition, it may be important in inflammation associated with severe trauma, where the drivers of inflammation are substantial and acute tissue damage, and extensive activation of the coagulation and haemostatic pathway. However, we believe that during acute responses initiated by inflammatory cytokines in a coordinated and controlled manner, and where timely and comprehensive resolution is the norm, platelet-mediated pathways of leukocyte trafficking are likely to be of lesser importance.

Other studies show that whole platelets can bind leukocytes, a process dependent upon platelet and/or leukocyte activation and linked to pathological conditions. ${ }^{46}$ Moreover, if PEV are mixed with isolated monocytes they are able to activate the leukocytes so that they show enhanced levels of recruitment to EC in vitro, although direct binding between PEV and leukocytes was not demonstrated in that study. ${ }^{47}$ Here, we show that monocytes preferentially accumulate PEV rather than whole platelets through an adhesive pathway reliant upon P-selectin. In the context of leukocyte recruitment to vascular EC, P-selectin supports a distinct form of rolling adhesion which is based on the transient nature of the bonds formed with PSGL-1 under conditions of shear. ${ }^{48}$ Here we propose that the P-selectin-PSGL-1 mediated interactions between PEV and leukocytes are also transient under the shear conditions of our assay and in flowing blood in vivo. However, on monocytes and neutrophils, PS in the PEV membrane acts to stabilise heterotypic adhesion upon interaction with membrane receptors on the leukocytes. In the case of $\mathrm{T}$ lymphocytes, which also possess abundant PSGL-1, the transient interactions formed with P-selectin under shear are not stabilised by PS, which has not been reported to bind $\mathrm{T}$ cells to our knowledge.

In fact, much of the data on heterotypic aggregate formation in human blood does not discriminate between platelets and PEV binding to leukocytes, and it is unclear which is being assessed. Studies that do report platelet
A

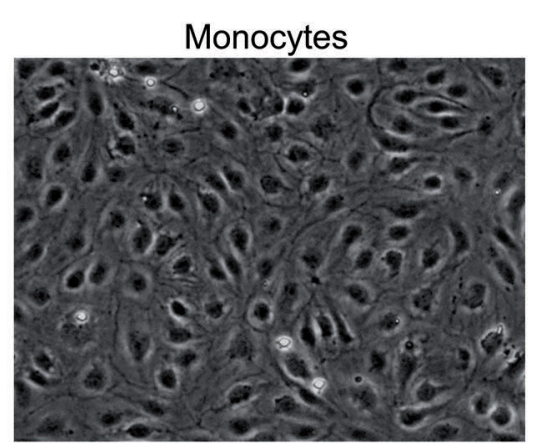

B

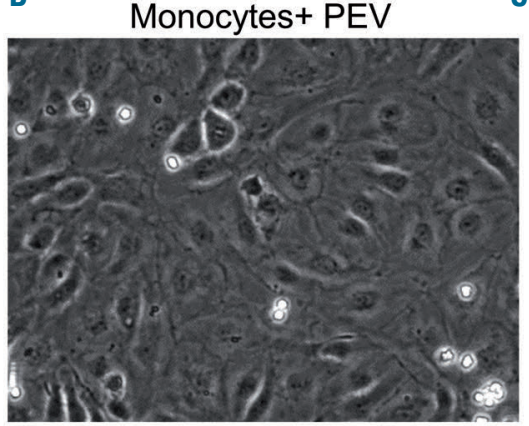

C Monocytes+ PEV+ Anti-GPIb

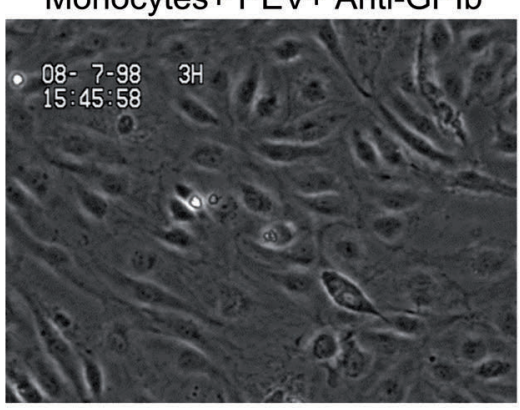

D

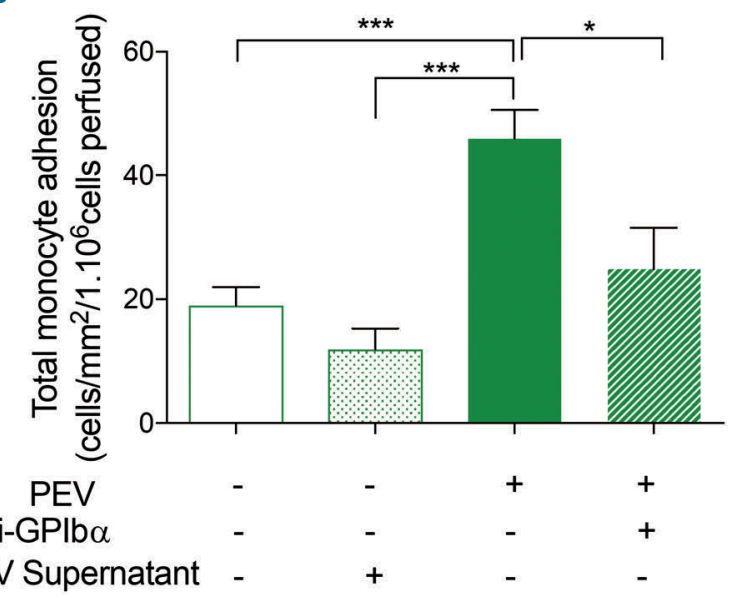

$E$

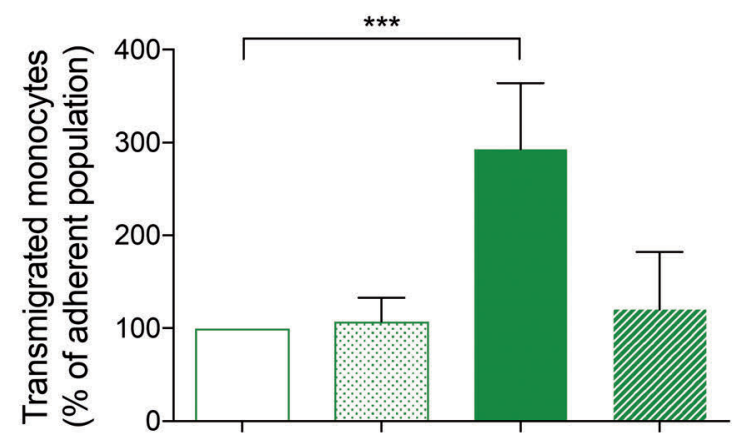

PEV

Anti-GPIb $\alpha$

PEV Supernatant -

Figure 6. GPIb $\alpha$ derived from platelet-derived extracellular vesicles supports monocytes recruitment on TGF- $\beta 1$ stimulated endothelial cells. (A-C) Representative pictures of monocytes (A), monocytes bearing CRP_XL (1 $\mu \mathrm{g} / \mathrm{mIL})$ generated-PEV (B) and monocytes bearing PEV with GPIb $\alpha$ blockade (clone $6 \mathrm{~B} 4,20 \mu \mathrm{g} / \mathrm{mL})(\mathrm{C})$, adhered on TGF- $\beta 1(10 \mathrm{ng} / \mathrm{mL})$ stimulated EC in flow conditions. (D, E) Total adhesion (D) and transmigration (E) of monocytes with or without PEV, GPIba blockade and filtered PEV through a $10 \mathrm{KDa}$ filters to remove PEV and leave potential soluble factors on TGF- $\beta 1$ stimulated EC in flow conditions, $n=3-5$. Data are mean \pm standard error of the mean (SEM). ${ }^{*} P \leq 0.05,{ }^{*} P \leq 0.01$ by ANOVA and Bonferroni post-test. 
A

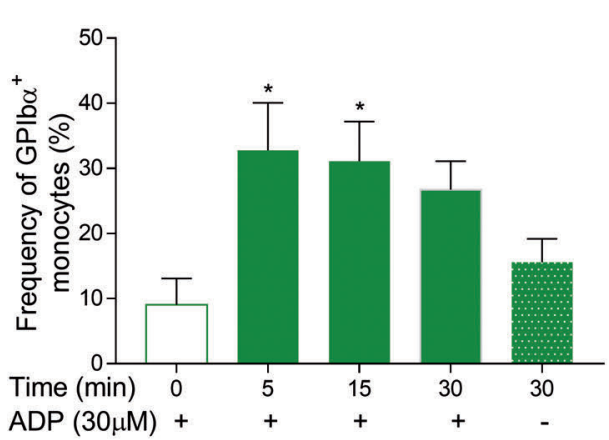

D

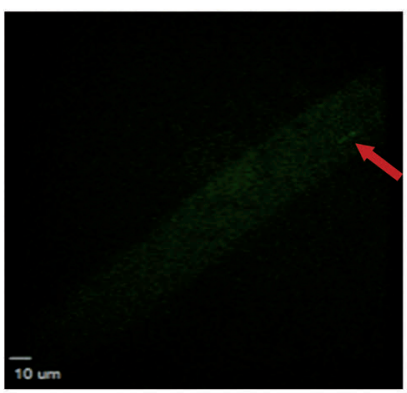

E

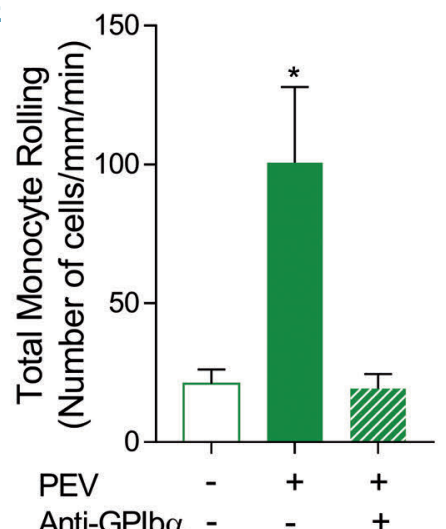

B

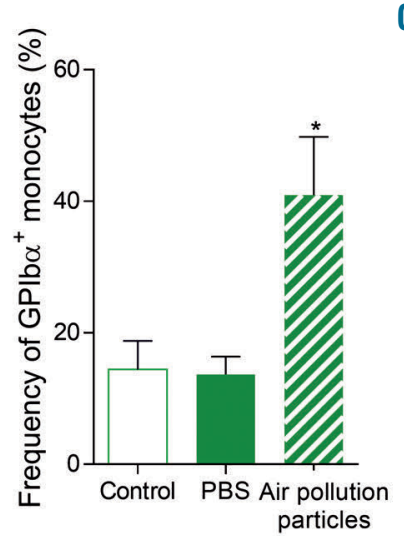

C

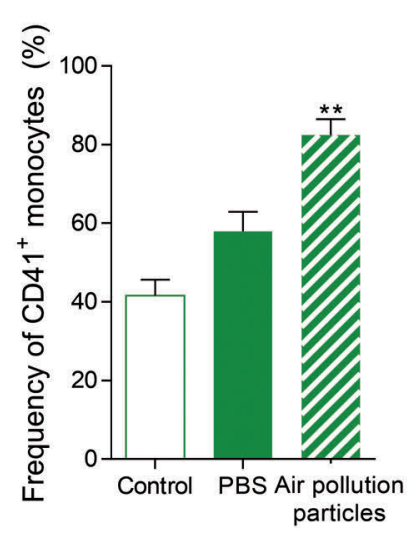

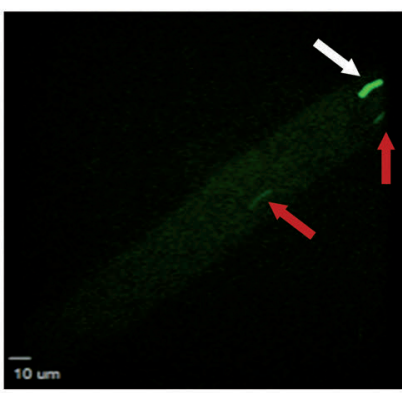

$\mathrm{F}$

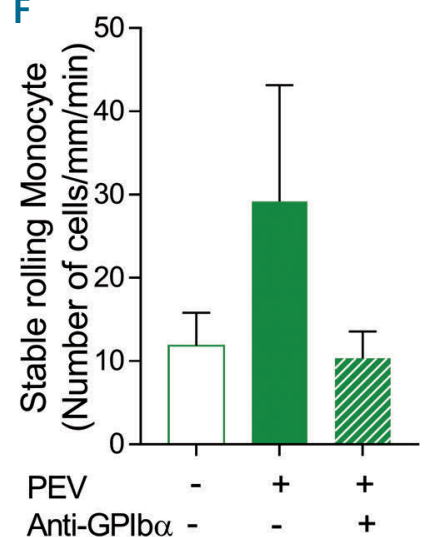

G

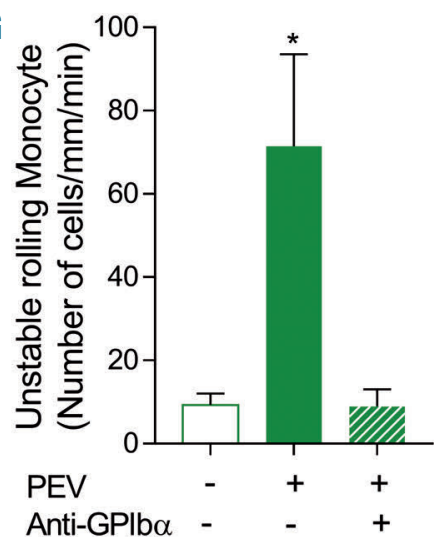

Anti-GPIba - - +
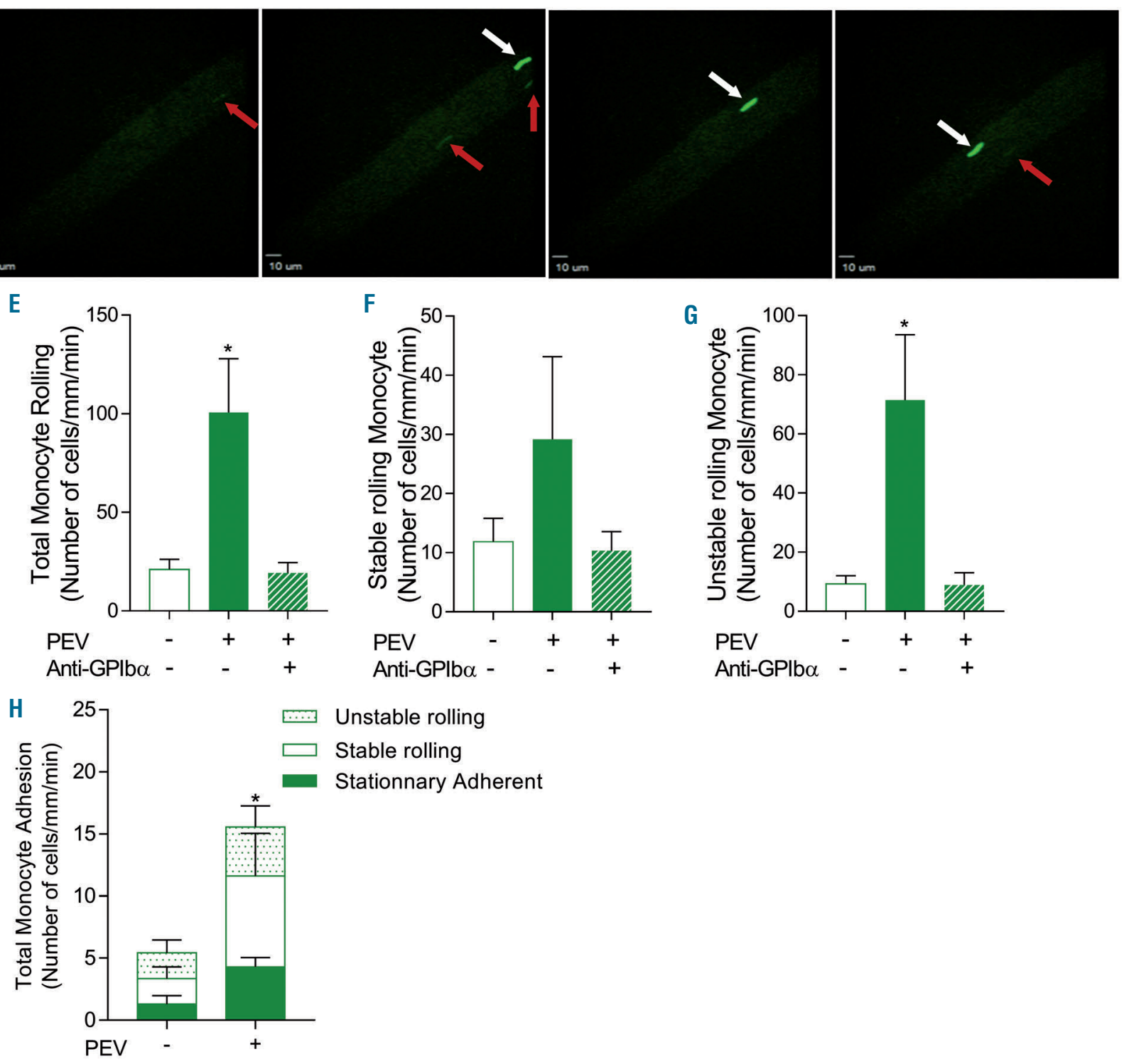

Figure 7. GPIb $\alpha$ from platelet-derived extracellular vesicles mediates recruitment of monocytes in vivo. (A) Percentage of GPIba ${ }^{+}$monocytes in whole murine blood stimulated with $30 \mu \mathrm{M} \mathrm{ADP}$ at $37^{\circ} \mathrm{C}$ under shear determined by flow cytometry, $\mathrm{n}=4$. (B) Percentage of GPIb $\alpha^{+}$and (C) CD41 monocytes in murine blood 48 hours after intratracheal instillation of air pollution particles $(400 \mu \mathrm{g}$ ) measured by flow cytometry, $\mathrm{n}=5-6$. (D) Representative images of the recruitment of human monocytes bearing GPIb $\alpha$ from mouse platelet-derived extracellular vesicles (PEV) to the TGF- $\beta 1(80 \mu \mathrm{g} / \mathrm{kg})$-stimulated vasculature in the cremaster muscle using intravital microscopy. Red arrows point to unstable rolling monocytes, white arrows to stable rolling monocytes. Total rolling $(E)$ stable rolling $(F)$ and unstable rolling (G) of human monocytes bearing GPIb $\alpha$ from mouse PEV with or without with GPIb $\alpha$ blockade (clone Xia.B2, $50 \mu \mathrm{g} / \mathrm{mL}$ ) adoptively transferred in the platelet depleted IL4R/GPIb $\alpha$-Tg mice measured by intravital microscopy of the TGF $\beta 1$-stimulated cremaster muscle, $n=3$. (H) Total adhesion and behaviors of human monocytes bearing mouse PEV adoptively transferred in western diet fed $A p o E$ mice measured by intravital microscopy of the right carotid artery, $n=3$. Data are mean \pm standard error of the mean (SEM). ${ }^{*} P \leq 0.05, * * P \leq 0.01$ compared to the 0 minute (min) control (A) or control (B, C) by analysis of variance (ANOVA) and Dunnett posttest and by unpaired $t$-test, by two-way ANOVA $(\mathrm{H})$. 
binding are routinely performed ex vivo under the nonphysiological condition of stasis in vitro, where the number of platelet-leukocyte aggregates formed is a direct function of the time of incubation. ${ }^{49}$ Thus, patient blood may have a greater propensity to form aggregates with platelets under static conditions ex vivo, but this probably does not reflect the situation in vivo. Such aggregation may be a surrogate endpoint for the degree of platelet and/or leukocyte activation present in patient blood. In support of this, the patterns of PEV associated with circulating monocytes that we report here are in strong accord with a recent report from Fendl et al. who analysed the effects of pre-analytical blood handling (which included the imposition of shear) on the association of extracellular vesicles with leukocytes. ${ }^{50}$

Interestingly, upon addition of purified PEV to whole blood, we observed rapid accumulation of GPIbo on monocytes, implying assimilation of PEV was extremely efficient. However, when a platelet activating agonist was added to whole blood the process was continuous and prolonged, leading to an incremental increase in GPIb $\alpha$ expression. The latter profile of accumulation of GPIb $\alpha$ likely reflects the dynamics of PEV formation and release by platelets in whole blood, implying that the rate-limiting step in this thrombo-inflammatory pathway is not $\mathrm{PEV}$-monocyte interaction, but rather the process of PEV release after platelet activation. In addition, accumulation of PEV was more prevalent in monocytes compared to neutrophils and lymphocytes. In a previous study, we observed different patterns of recruitment, migration and reverse migration in vitro between classical and non-classical/intermediate monocytes. ${ }^{43}$ We characterised a novel process of crosstalk mediated by cytokines between the two subsets that allowed a balanced regulation of endothelial cell activation. Other studies have shown that changes in proportional representation of monocyte subsets in the circulation are associated with vascular diseases. ${ }^{51,52}$ However, in this study we observed no preferential binding of PEV between classical and nonclassical/intermediate monocytes, which was consistent with similar levels of PSGL1 expression exhibited by all subsets.

GPIb $\alpha$ is an adhesion receptor mediating a specialised form of platelet recruitment during haemostasis. Bonds forming between GPIb $\alpha$ and VWF exhibit high on rates, meaning that adhesion can occur between rapidly flowing
A

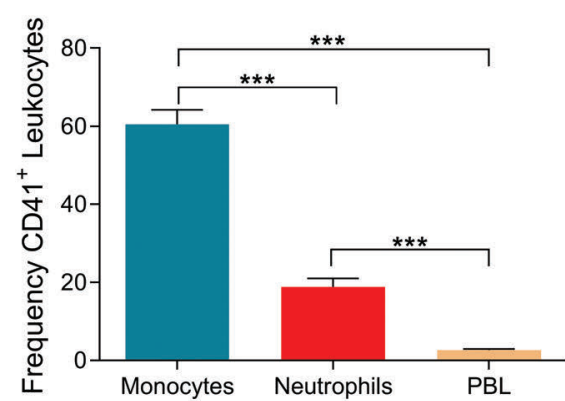

C

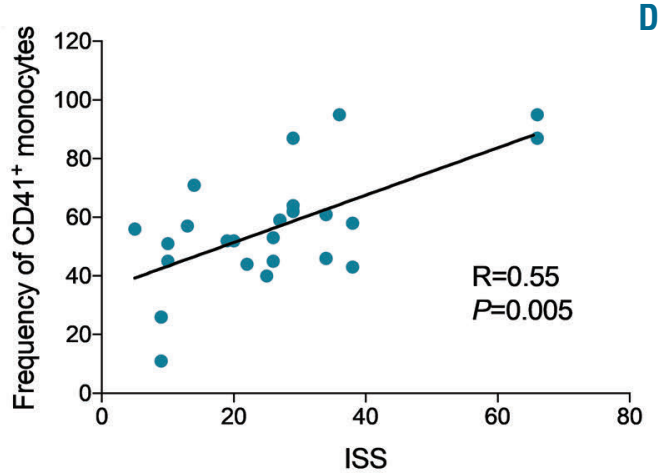

E

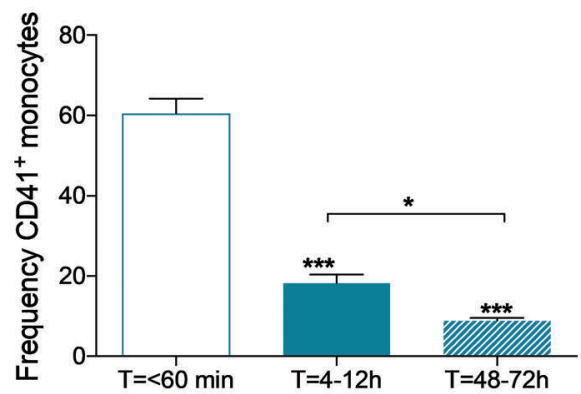

D
B
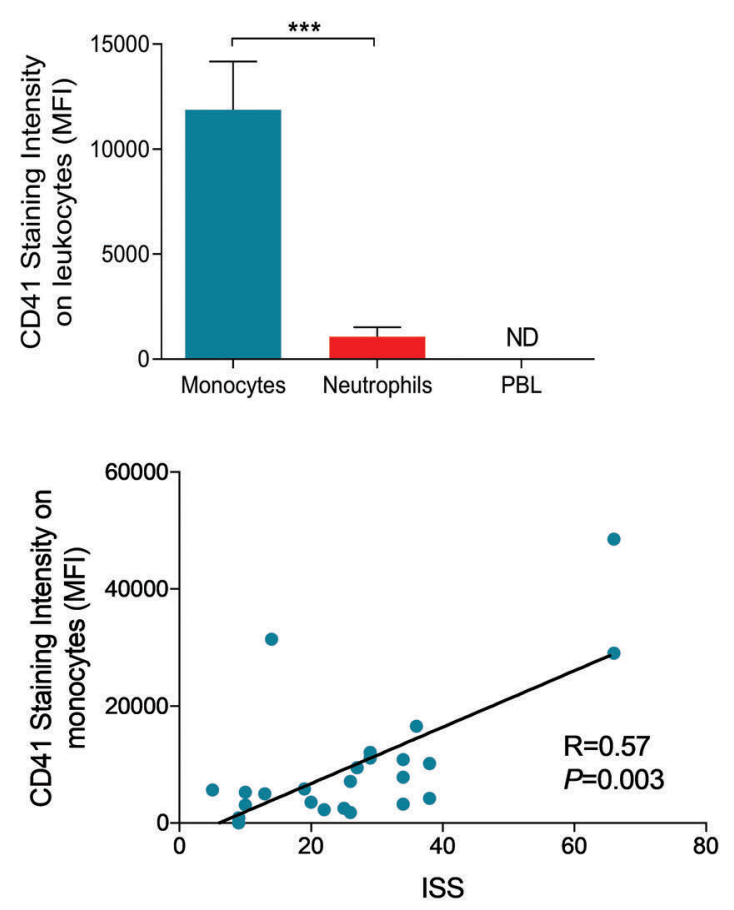

Figure 8. Monocytes accumulate platelet-derived extracellular vesicles (PEV) derived marker CD41 in trauma patients. (A, B) Percentage (A) and median fluorescent intensity (MFI) (B) of $\mathrm{CD} 41^{+}$monocytes, neutrophils and lymphocytes $(P B L)$ in whole blood in patients at time point $(T)<60$ minutes (min) after trauma, $n=31-35$. ND: non detectable for lymphocytes. (C, D) Percentage (C) and MFI (D) of CD41 ${ }^{+}$monocytes in whole blood in patients at $\mathrm{T}<60$ min after trauma plotted against Injury Severity Score (ISS), $n=28$. (E) Percentage of $C D 41^{+}$monocytes in whole blood in trauma patients at $\mathrm{T}<60$ minutes, $4-12$ hours and $48-72$ hours, $n=33$. Data are mean \pm standard error of the mean (SEM) $(A, B$ and $E)$. $* P \leq 0.05, * * * P \leq 0.001$ by ANOVA and Bonferroni post-test $(\mathrm{A}, \mathrm{E})$, Mann Whitney t-test $(B)$ and linear regression $(C, D)$. 
platelets and the substrate..$^{15,53}$ However, these bonds also exhibit high off rates. Thus, under conditions of shear stress (i.e. blood flow) the rapid formation and dissolution of bonds supports rolling adhesion. ${ }^{15,53}$ We observed that monocytes bearing GPIb $\alpha$ also rolled on purified VWF. However, on EC bearing VWF, monocytes were rapidly activated, which is consistent with our previous observations on the activity of EC derived stimuli such as C-C chemokine ligand 2 (CCL2). ${ }^{15}$

Trogocytosis is the phenomenon by which lymphocytes extract surface molecules from antigen presenting cells through transfer of plasma membrane at the immunological synapse. ${ }^{54}$ This process has been observed for $\mathrm{T}, \mathrm{B}$ and NK cells and neutrophils ${ }^{54,55}$ and is a fast and efficient means of transferring molecules involved in the regulation of immune functions. ${ }^{54}$ We cannot completely exclude that monocytes in whole blood do not bind whole platelets and acquire GPIbo and CD41 via a trogocytosis like process, although a synapse like structure has not been reported in this context to our knowledge. However, both trogocytosis and PEV accumulation by monocytes require activation dependent cytoskeletal rearrangement to achieve the transfer of membrane cargo that alters the function of the recipient cells. Thus, the processes may not be unrelated in their mechanisms of initiation and progression. However, trogocytosis does appear to be specific to the immunological synapse or related structures. ${ }^{54,56}$ Here however, we have shown that purified labelled PEV bind to isolated monocytes or monocytes in whole blood with the same dynamics as agonist stimulated systems. This clearly demonstrates that a trogocytosis like process is not required for accumulation of PEV once they have been generated by platelet activation (Figure 3). Our colleagues have also shown that PEV levels increase dramatically after trauma and thus are likely to be the source of GPIba found on leukocytes in trauma patients. ${ }^{57}$

Using intravital microscopy we observed GPIbo-dependent recruitment of PEV-treated monocytes to the vasculature. Interestingly, the short-lived adhesive interactions, here termed 'transient rolling' which did not result in prolonged monocyte localisation and activation at the vessel wall, have previously been shown to have physiological roles. Thus, under steady-state conditions (non-inflamed), circulating platelets expressing GPIb $\alpha$ are able to interact transiently with sinusoidal Kupffer cells in the liver via surface-expressed VWF, interactions which are important for host defence, as they facilitate uptake and disposal of bacteria by liver resident macrophages (Kupffer cells). ${ }^{58}$ In the context of CVD, we showed that induction of pulmonary inflammation with pollution nanoparticles, a known risk factor for thrombo-inflammatory disease associated with atherosclerosis ${ }^{59}$ induced the formation of circulating monocyte-PEV aggregates. Moreover, such aggregates showed a significantly enhanced capacity to bind to the artery wall in the $A p o E^{-/}$mouse after induction of disease by feeding a high fat western diet. Thus, we propose that the transfer of platelet cargo to monocytes by PEV can contribute to the progression of plaque formation by promoting the recruitment of inflammatory monocytes. It would be interesting to investigate the functional and phe- notypical changes induced by binding and internalisation of PEV by monocytes. In this study we did not observe major changes in integrin expression as a marker of activation. However, we do not exclude changes in monocyte activation and/or function relevant to vascular disease over longer periods of interaction.

The paradigm discussed above may provide a novel thrombo-inflammatory mechanism for the continuous low levels of monocyte delivery in chronic inflammatory conditions such as atherosclerosis. However, our Golden Hour data suggest that acquisition of this pathway of monocyte recruitment could also lead to the clearance of monocytes from the blood during acute and severe trauma. Indeed, in this injured patient cohort, PEV counts increase in the circulation ${ }^{57}$ and monocytes rapidly acquired CD41-derived from PEV (1 hour after trauma) which we believe led to their clearance from the circulation, as frequency of $\mathrm{CD} 41^{+}$monocyte numbers are lower 4 hours after trauma. This may be due to clearance by the reticulo-EC system, or alternatively by the expedited recruitment to damaged and inflamed tissues, or indeed a combination of both. Whatever the pathway of their removal from the circulation, we speculate that the rapid clearance of immune cells from the circulation may exacerbate cell turnover and result in immune suppression and the increased risk of septic complications. In addition, monocytes bearing pro-coagulant PEV could also contribute to the initiation and propagation of disseminated intravascular coagulation (DIC) which is a potential and serious complication of traumatic injury. ${ }^{27}$

In conclusion, we believe that this new paradigm for leukocyte recruitment is an important step in understanding the contribution of platelets to thrombo-inflammatory pathology. By acquiring GPIb $\alpha$ in the circulation, monocytes may be provided with a means of interacting with the vessel wall, which is ordinarily restricted to platelets during haemostasis. In chronic diseases such as atherosclerosis, this process may occur with a low frequency over protracted periods of time. Nevertheless, the dynamic nature of PEV-monocyte interaction demonstrated in this study implies that such routes of thrombo-inflammation may be major contributors to pathology.

\section{Funding}

This work was supported by a British Heart Foundation (BHF) programme grant (RG/12/7/29693 to GER), a BHF Chair (CH/03/003 to SPW), a Royal Society Dorothy Hodgkin research fellowship (DH160044 to $M C$ ) and a BHF studentship (FS/14/42/30956, GER). This work was also supported by grants from the NIHR Surgical Reconstruction Microbiology Research Centre (SRMRC) (JL,JH) and the Scar Free foundation (JL, PH). The NIHR-SRMRC is a partnership between University Hospitals Birmingham NHS Foundation Trust, the University of Birmingham and the Royal Centre for Defence Medicine. The views expressed are those of the author(s) and not necessarily those of the NHS, the NIHR or the Department of Health. $A B$ is supported by British Heart Foundation Senior Basic Science Research Fellowship (FS/19/30/34173). This work was also supported by a BHF Accelerator Award (AA/18/2/34218). 


\section{References}

1. Kansas GS. Selectins and their ligands: current concepts and controversies. Blood. 1996;88(9):3259-3287.

2. Ley K, Laudanna C, Cybulsky MI, Nourshargh S. Getting to the site of inflammation: the leukocyte adhesion cascade updated. Nat Rev Immunol. 2007;7(9):678689

3. Liu Y, Shaw SK, Ma S, Yang L, Luscinskas FW, Parkos CA. Regulation of leukocyte transmigration: cell surface interactions and signaling events. J Immunol. 2004;172(1):713 .

4. Springer TA. Traffic signals on endothelium for lymphocyte recirculation and leukocyte emigration. Annu Rev Physiol. 1995;57:827872.

5. McEver RP. Adhesive interactions of leukocytes, platelets, and the vessel wall during hemostasis and inflammation. Thromb Haemost. 2001;86(3):746-756.

6. Cooper D, Russell J, Chitman KD, Williams MC, Wolf RE, Granger DN. Leukocyte dependence of platelet adhesion in postcapillary venules. Am J Physiol Heart Circ Physiol. 2004;286(5):H1895-900.

7. Kuroda T, Shiohara E. Leukocyte and platelet depletion protects the liver from damage induced by cholestasis and ischemia-reperfusion in the dog. Scand J Gastroenterol. 1996;31(2):182-190

8. Lefer AM, Campbell B, Scalia R, Lefer DI. Synergism between platelets and neutrophils in provoking cardiac dysfunction after ischemia and reperfusion: role of selectins. Circulation. 1998;29;98(13):13221328.

9. Nishijima K, Kiryu J, Tsujikawa A, et al. In vivo evaluation of platelet--endothelial interactions after transient retinal ischemia. Invest Ophthalmol Vis Sci. 2001;42(9):21022109.

10. Salter JW, Krieglstein CF, Issekutz AC, Granger DN. Platelets modulate ischemia/reperfusion-induced leukocyte recruitment in the mesenteric circulation. Am I Physiol Gastrointest Liver Physiol. 2001;281(6):G1432-1439.

11. Burger PC, Wagner DD. Platelet P-selectin facilitates atherosclerotic lesion development. Blood. 2003;101(7):2661-2666.

12. Huo Y, Schober A, Forlow SB, et al. Circulating activated platelets exacerbate atherosclerosis in mice deficient in apolipoprotein E. Nat Med. 2003;9(1):61-67.

13. Massberg S, Brand K, Gruner S, et al. A critical role of platelet adhesion in the initiation of atherosclerotic lesion formation. J Exp Med. 2002;196(7):887-896.

14. Sachais BS, Turrentine T, Dawicki McKenna JM, Rux AH, Rader D, Kowalska MA. Elimination of platelet factor 4 (PF4) from platelets reduces atherosclerosis in $\mathrm{C} 57 \mathrm{Bl} / 6$ and apoE-/- mice. Thromb Haemost. 2007;98(5):1108-1113

15. Kuckleburg CJ, Yates CM, Kalia N, et al. Endothelial cell-borne platelet bridges selectively recruit monocytes in human and mouse models of vascular inflammation. Cardiovasc Res. 2011;91(1):134-141.

16. Elalamy I, Chakroun T, Gerotziafas GT, et al. Circulating platelet-leukocyte aggregates: a marker of microvascular injury in diabetic patients. Thromb Res. 2008;121(6):843-848.

17. Goncalves R, Zhang X, Cohen H, Debrabant A, Mosser DM. Platelet activation attracts a subpopulation of effector monocytes to sites of Leishmania major infection. J Exp Med. 2011;208(6):1253-1265.
18. Harding SA, Sommerfield AJ, Sarma J, et al. Increased CD40 ligand and platelet-monocyte aggregates in patients with type 1 diabetes mellitus. Atherosclerosis. 2004;176(2):321-325

19. Joseph JE, Harrison P, Mackie IJ, Isenberg DA, Machin SJ. Increased circulating platelet-leucocyte complexes and platelet activation in patients with antiphospholipid syndrome, systemic lupus erythematosus and rheumatoid arthritis. Br J Haematol. 2001;115(2):451-459.

20. McCabe DJ, Harrison P, Mackie IJ, et al. Platelet degranulation and monocyteplatelet complex formation are increased in the acute and convalescent phases after ischaemic stroke or transient ischaemic attack. Br J Haematol. 2004;125(6):777-787.

21. Parimon T, Li Z, Bolz DD, et al. Staphylococcus aureus alpha-hemolysin promotes platelet-neutrophil aggregate formation. J Infect Dis. 2013;208(5):761-770

22. Tekelioglu Y, Uzun H. Circulating plateletleukocyte aggregates in patients with inflammatory bowel disease. J Chin Med Assoc. 2013;76(4):182-185.

23. Gkaliagkousi E, Corrigall V, Becker S, et al. Decreased platelet nitric oxide contributes to increased circulating monocyte-platelet ggregates in hypertension. Eur Heart J. 2009;30(24):3048-3054.

24. Nomura S, Kanazawa S, Fukuhara S. Effects of efonidipine on platelet and monocyte activation markers in hypertensive patients with and without type 2 diabetes mellitus. J Hum Hypertens. 2002;16(8):539-547.

25. Wrigley BJ, Shantsila E, Tapp LD, Lip GY. Increased formation of monocyte-platelet aggregates in ischemic heart failure. Circ Heart Fail. 2013;6(1):127-135.

26. Lippi G, Montagnana M, Salvagno GL, et al. Risk stratification of patients with acute myocardial infarction by quantification of circulating monocyte-platelet aggregates. Int Cardiol. 2007:115(1):101-102

27. Gando S, Otomo Y. Local hemostasis, immunothrombosis, and systemic disseminated intravascular coagulation in trauma and traumatic shock. Crit Care. 2015;19:72.

28. Ogura H, Kawasaki T, Tanaka H, et al. Activated platelets enhance microparticle formation and platelet-leukocyte interaction in severe trauma and sepsis. I Trauma. 2001:50(5):801-809.

29. Raposo G, Stoorvogel W. Extracellular vesicles: exosomes, microvesicles, and friends. J Cell Biol. 2013;200(4):373-383.

30. Cloutier N, Tan S, Boudreau LH, et al. The exposure of autoantigens by microparticles underlies the formation of potent inflammatory components: the microparticle-associated immune complexes. EMBO Mol Med. 2013;5(2):235-249.

31. Holme PA, Rosger M, Solum NO, Brosstad F, Larsen AM, Hovig T. Glycoprotein IIb-IIIa on platelet-derived microparticles, and microparticle structures studied by electron microscopy, confocal laser microscopy and crossed radio-immunoelectrophoresis. Platelets. 1996;7(4):207-214

32. Nomura S, Tandon NN, Nakamura T, Cone J, Fukuhara S, Kambayashi J. High-shearstress-induced activation of platelets and microparticles enhances expression of cell adhesion molecules in THP-1 and endothelial cells. Atherosclerosis. 2001;158(2):277287.

33. Shai E, Rosa I, Parguina AF, Motahedeh S, Varon D, Garcia A. Comparative analysis of platelet-derived microparticles reveals differences in their amount and proteome depending on the platelet stimulus. Proteomics. 2012;76 Spec No.:287-296.

34. Boudreau LH, Duchez AC, Cloutier N, et al Platelets release mitochondria serving as substrate for bactericidal group IIA-secreted phospholipase A2 to promote inflammation. Blood. 2014;124(14):2173-2183.

35. Aatonen MT, Ohman T, Nyman AT, Laitinen S, Gronhilm M, Siljander PRM Isolation and characterisation of plateletderived extracellular vesicles. J Extracell Vesicles. 2014;3.

36. Ponomareva AA, Nevzorova TA, Mordakhanova ER, Andrianova IA, Litvinov RI. Structural characterisation of platelets and platelets microvesicles. Cell Tissue Biol. 2016; 10(3):217-226.

37. Boilard E, Duchez AC, Brisson A. The diversity of platelet microparticles. Curr Opin Hematol. 2015;22(5):437-444.

38. Varon D, Hayon Y, Dashevsky O, Shai E. Involvement of platelet derived microparticles in tumor metastasis and tissue regeneration. Thromb Res. 2012;130 Suppl 1:S9899.

39. Ogata $N$, Imaizumi $M$, Nomura $S$, et al Increased levels of platelet-derived microparticles in patients with diabetic retinopathy. Diabetes Res Clin Pract. 2005;68(3):193-201

40. Nomura S, Suzuki M, Katsura K, et al Platelet-derived microparticles may influence the development of atherosclerosis in diabetes mellitus. Atherosclerosis. 1995;116 (2):235-240

41. Feng B, Chen Y, Luo Y, Chen M, Li X, Ni Y. Circulating level of microparticles and their correlation with arterial elasticity and endothelium-dependent dilation in patients with type 2 diabetes mellitus. Atherosclerosis. 2010;208(1):264-269.

42. Biasucci LM, Porto I, Di Vito L, et al. Differences in microparticle release in patients with acute coronary syndrome and stable angina. Circ J. 2012;76(9):2174-2182.

43. Chimen M, Yates CM, McGettrick HM, et al. Monocyte Subsets Coregulate Inflammatory Responses by Integrated Signaling through TNF and IL- 6 at the Endothelial Cell Interface. J Immunol. 2017;198(7):2834-2843

44. Daaboul GG, Gagni P, Benussi L, et al. Digital Detection of Exosomes by Interferometric Imaging. Sci Rep. 2016; 6:37246.

45. Mause SF, von Hundelshausen P, Zernecke A, Koenen RR, Weber C. Platelet microparticles: a transcellular delivery system for RANTES promoting monocyte recruitment on endothelium. Arterioscler Thromb Vasc Biol. 2005;25(7):1512-1518

46. van Gils JM, Zwaginga JJ, Hordijk PL. Molecular and functional interactions among monocytes, platelets, and endothelial cells and their relevance for cardiovascuar diseases. J Leukoc Biol. 2009;85(2):195204.

47. Barry OP, Pratico D, Lawson JA, FitzGerald GA. Transcellular activation of platelets and endothelial cells by bioactive lipids in platelet microparticles. J Clin Invest. 1997:99(9):2118-2127.

48. Ley K. The role of selectins in inflammation and disease. Trends Mol Med. 2003:9(6):263-268

49. Harding SA, Din JN, Sarma J, et al. Flow cytometric analysis of circulating platelet monocyte aggregates in whole blood: methodological considerations. Thromb Haemost. 2007;98(2):451-456.

50. Fendl B, Weiss R, Fischer MB, Spittler A 
Weber V. Characterization of extracellular vesicles in whole blood: Influence of preanalytical parameters and visualization of vesicle-cell interactions using imaging flow cytometry. Biochem Biophys Res Commun. 2016;478(1):168-173.

51. Shantsila E, Wrigley B, Tapp L, et al. Immunophenotypic characterization of human monocyte subsets: possible implications for cardiovascular disease pathophysiology. J Thromb Haemost. 2011;9(5):10561066.

52. Yang J, Zhang L, Yu C, Yang XF, Wang $H$. Monocyte and macrophage differentiation: circulation inflammatory monocyte as biomarker for inflammatory diseases. Biomark
Res. 2014;7;2(1):1.

53. Andre P, Denis CV, Ware J, et al. Platelets adhere to and translocate on von Willebrand factor presented by endothelium in stimulated veins. Blood. 2000;96(10):3322-3328.

54. Ahmed KA, Munegowda MA, Xie Y, Xiang J. Intercellular trogocytosis plays an important role in modulation of immune responses. Cell Mol Immunol. 2008;5(4):261-269.

55. Valgardsdottir R, Cattaneo I, Klein C, Introna M, Figliuzzi M, Golay J. Human neutrophils mediate trogocytosis rather than phagocytosis of CLL B cells opsonized with anti-CD20 antibodies. Blood. 2017;129(19): 2636-2644.

56. Dopfer EP, Minguet S, Schamel WW. A new vampire saga: the molecular mechanism of T cell trogocytosis. Immunity. 2011;35(2): 151-153.

57. Kuravi SJ, Yates CM, Foster $M$, et al Changes in the pattern of plasma extracellular vesicles after severe trauma. PLoS One. 2017;12(8):e0183640.

58. Wong CH, Jenne CN, Petri B, Chrobok NL, Kubes P. Nucleation of platelets with bloodborne pathogens on Kupffer cells precedes other innate immunity and contributes to bacterial clearance. Nat Immunol. 2013;14 (8):785-792.

59. Brook RD, Rajagopalan S. Particulate matter air pollution and atherosclerosis. Curr Atheroscler Rep. 2010;12(5):291-300. 